\title{
CONNAISSANCE ET MÉTAPHORE
}

\author{
Judith SCHLANGER
}

RÉsumé: Cette étude consacrée à la dimension heuristique des métaphores cherche à comprendre le rôle qu'elles jouent dans l'activité générale de la pensée. Le rôle des métaphores est particulièrement visible à l'état naissant de la pensée connaissante, au point où son exploration trouve dans l'étoffe du langage les moyens de conceptualiser le nouveau. L'exemple de Savigny, de Bacon et de Kant montre comment l'intuition métaphorique et la reprise culturelle des schèmes métaphoriques ont une fonction intellectuelle féconde.

MoTs clÉs : pensée, connaissance, heuristique, métaphores.

SUMmaRY : This paper deals with the heuristic dimension of metaphors and their role within thought and knowledge. The function of metaphors is especially salient at the moment of birth, when language enables thought to grasp and conceptualize a new territory. Savigny, Bacon, Kant illustrate the fruitful intellectual and cognitive function of metaphorical intuition, as well as of the cultural retention and replay of some important metaphorical figures and schemes.

KEYworDS : thought, knowledge, heuristics, metaphors.

Revue de synthèse : $4^{e}$ S. $N^{0} 4$, oct.-déc. 1995, p. 579-592. 
ZuSAMMENFASSUNG : In diesem Artikel geht es um die heuristische Dimension von Metaphern und um deren Rolle beim Denken und Erkennen. Die Rolle der Metapher erweist sich vor allem am Anfang des erkennenden Denkens, wenn das Denken durch die Sprache in die Lage versetzt wird, neue Begriffe zu entwickeln. Am Beispiel von Savigny, Bacon und Kant wird gezeigt, wie fruchtbar die metaphorische Intuition und das in der kulturellen Entwicklung feststellbare Weiterbestehen metaphorischer Formen im Erkenntnisproze $\beta$ ist.

STICHWÓRTER : Gedanke, Erkenntnis, Heuristik, Metaphern.

Judith SCHLANGER, née en 1936, enseigne à l'Université hébraïque de Jérusalem. Elle a publié en France de nombreux livres sur la pensée connaissante, son langage, son invention et sa mémoire.

Adresse: The Hebrew University of Jerusalem, Faculty of Humanities, Mount Scopus, Jerusalem, Israel. 
Les métaphores remplissent plusieurs sortes de fonctions intellectuelles par rapport au raisonnement : des fonctions argumentatives, mais aussi des fonctions directement cognitives. Les fonctions idéologiques et polémiques sont particulièrement voyantes. Le langage socio-politique des publicistes d'il y a un siècle en est un bon exemple, ce langage qui reprend la vieille métaphore classique de la réflexion politique, la métaphore du corps politique objet de l'attention quasi médicale des gouvernants, à la lumière du récent discours pastorien. Lorsqu'on analyse ce à quoi on s'oppose comme une maladie du pays ou de l'Etat, lorsqu'on décrit l'obstacle ou l'adversaire comme un cancer, une gangrène, une infection, comme une pourriture locale qu'il faut amputer ou un désordre organique général qu'il faut réguler, qu'espère-t-on gagner? On espère entraîner l'adhésion intuitive du public et mobiliser son désir polémique d'intervention. On se place dans un champ verbal et intuitif à la fois traditionnel et actuel, qui parait fonder le débat, et que le débat enrichit.

Ces fonctions idéologiques, dont je n'ai évoqué ici qu'un exemple, sont extrêmement importantes, mais elles n'épuisent pas la dimension intellectuelle du métaphorique. Les métaphores sont aussi des sources d'énoncés qui permettent d'avancer dans la pensée, de commencer à parler de ce qu'on ne connaît pas encore, et d'ouvrir par là même un nouvel espace de diction, d'exploration et d'interprétation.

Comment comprendre que des métaphores jouent un rôle dans l'avancée de la connaissance, et que des déplacements de langage contribuent à l'invention intellectuelle? J'explorerai ici cette dimension heuristique des métaphores.

\section{LA PENSÉE CONNAISSANTE}

Soit des notions ou des constellations de notions déplacées hors de leur terrain d'origine et employées ailleurs, dans un autre champ de pertinence. De tels transferts ne sont pas rares : ils sont, au contraire, un accompagnement mobile mais abondant de la réflexion. Leur démultiplication même fait d'eux un phénomène important de la connaissance active. Le paysage général de ces transferts métaphoriques apparaît mieux si l'on considère l'activité de connaissance en termes très larges, c'est-à-dire si, au lieu de penser à des catégories disciplinaires ou même à des catégories englobantes comme la science ou la philosophie, on s'interroge sur la pensée connaissante en général. 
Pour caractériser (rapidement) cette activité générale de pensée, on notera tout d'abord que c'est une pensée liée au langage. Il est vrai que toute une polémique s'est développée sur ce.point depuis un bon siècle : existe-t-il une pensée symbolique abstraite hors langage? Oui, a-t-on dit quelquefois, soit en insistant sur l'intellect mathématique et musical, soit en plaçant au centre l'intellect plastique et spatial. Je propose de contourner ici cette discussion, pour considérer avant tout l'intellect conceptuel et donc verbal. Même s'il n'est pas le seul à chercher et à gagner le savoir, il n'en est pas moins considerable, puisqu'il est comme le nom premier de l'intelligence. L'intellect langagier, celui qui cherche à s'orienter dans la connaissance d'une façon discursive, englobe à la fois les spéculations les plus abstraites et la masse de la pensée commune; il se rapporte à la pointe agile de la conscience vive comme à la mémoire scolaire ou professionnelle des efforts dépensés; il est la première évidence de la réflexion : on réfléchit dans le langage, avec lui, à travers lui.

La pensée connaissante apparaît donc comme un territoire immense, très riche en productions particulièrement complexes, en raisonnements, en discours, en exposés d'idées où positions et propositions se développent et s'argumentent. On retrouve ici le champ de la communication rationnelle et de l'argumentation, ce champ exploré par Chaïm Perelman '; et on perçoit aussi comment il est possible que les phénomènes métaphoriques, qui sont par excellence d'ordre verbal, jouent un rôle proprement intellectuel dans l'entreprise de connaître.

Par le langage, la pensée connaissante abstraite, theoricienne, professionnelle, reste reliée à l'intellection commune et à l'intelligence courante. Assurément, la pensée abstraite est une pensée séparée qui se gagne un régime à part; elle se dégage du substrat commun et elle en sort. Mais ce dont elle est sortie, elle ne l'a pas radicalement quitté, pour la raison simple que le penseur, lui, n'est pas radicalement coupé de la personne vivante et banale qu'il est aussi la plupart du temps.

Cet ancrage de l'intellect abstrait dans l'intelligence commune est particulièrement important au moment de la naissance de la pensée. Ici conception et conceptualisation vont de pair, tout comme initiation et reprise, fondation et transformation. Tout se passe comme si le moment de la naissance avait un régime spécial qui éclaire à sa façon tout le phénomène de la pensée. La pensée abstraite émerge de son substrat psychique, de ce que Bachelard nommait l'homme nocturne ou l'homme pensif, celui qui rêve devant un feu et se laisse bercer dans l'autobus. Et la pensée professionnelle émerge de son substrat culturel et de sa mémoire. Mémoire

1. Chaïm Perelman et Lucie Olbrechts-Tyteca, Traité de l'argumentation, $5 e$ éd., Bruxelles, Éditions de l'université de Bruxelles, 1988. 
confuse, plurielle et riche : nous savons (mal) beaucoup de choses, nous circulons dans beaucoup de domaines, nous avons des ressources que nous ne nous sentons pas posséder, nous sommes plus riches, plus souples, moins rigoureux que nous ne pensons.

Si on regarde la pensée connaissante à l'état naissant, en observant, par exemple, les épisodes célèbres et les cas canoniques auxquels se plait l'histoire des sciences, on perçoit combien le discours abstrait trouve de ressources dans la parole culturelle dont il est en train de s'abstraire. L'abstrait se dégage du culturel et s'en nourrit, en retenant les schèmes et les notions métaphoriques dont il a besoin pour avancer. Or cet état naissant n'est pas seulement un stade initial traversé une fois pour toutes. Plus ou moins prononcé, plus ou moins mêlé, il ne cesse pas d'accompagner l'activité intellectuelle de connaissance : il est son état instable et sa fécondité.

Sa fécondité, au sens où, pour la réflexion, voir et dire se confondent. Pour commencer, pour avancer, pour penser, la pensée connaissante doit baliser et anticiper. Pour concevoir un point de vue différent, une question neuve, un sujet de recherche inédit, voire l'emplacement d'une nouvelle discipline, la pensée l'appréhende en commençant par en parler: elle nomme ce qui n'existe pas encore, elle désigne ce qui n'est pas encore connaissable, et d'une certaine façon elle le caractérise, pour accrocher à quelques catégories descriptives ses premières promesses d'explication ${ }^{2}$.

En ce point, l'exploration est purement déclarative, au sens où nommer revient à prendre possession de la suite possible. Ce qui se trouve énoncé, même très provisoirement et très approximativement, est déjà sorti de l'impensable. Stagflation, New Frontier, atomes, chaos: ces poteaux ne peuvent pas tenir tels quels. Ils appellent un travail indispensable : celui de l'élaboration de la réflexion et de l'épreuve du réel. Les rubriques ne peuvent pas subsister dans leur première fragilité intuitive et verbale; mais sans les termes qui ont posé leur première esquisse, comment la préoccupation prendrait-elle forme, comment la notion commencerait-elle à exister?

Certes, le rapport entre le vocable et le savoir n'est pas toujours le même, loin de là; ne serait-ce que parce que la désignation ne fạit pas le savoir, les premières problématiques peuvent s'effondrer en route et, de toute façon, dans l'ensemble de ce qui est proposé, tout ne tient pas, ne subsiste pas et ne réussit pas. Il est clair qu'il ne suffit pas de planter un drapeau. Cependant (on le voit bien à tous les niveaux, du journalistique au théorique), la conceptualisation est liée à la diction, et demande d'entrée de jeu des schèmes verbaux, des avancées discursives, qui donnent forme et direction à l'intuition.

2. Judith Schlanger, Penser la bouche pleine, Paris, Fayard, 1983, p. 13-23; ID., L'Invention intellectuelle, Paris, Fayard, 1983, p. 181-206. 
Autant ces schèmes verbaux sont nécessaires à la communication, puisqu'ils permettent de fixer et d'orienter l'attention, autant ils sont d'abord nécessaires à cette communication de soi à soi qui est la réflexion même et l'exercice du jugement. L'anticipation heuristique gagne l'altérité et la rend pensable en la plaçant dans le jeu du sens, qui est aussi le tissu du langage. Pour que la pensée inventive soit à la fois compréhensible pour elle-même et communicable, la parole inventive doit être référée. Peu importe en ce point si elle cherche à enrichir et à moduler son cadre conceptuel ou si elle entend fonder un cadre notionnel neuf; si elle entend poursuivre ou commencer : dans tous les cas, le langage demande à la fois la connexion et l'écart, l'altérité dans l'identité; ou encore, pour reprendre les catégories de la Rhétorique d'Aristote, le plaisir de la surprise et le plaisir des repères.

Mais ce que le langage demande, par lui-même il le procure. C'est le plein du langage intellectuel-culturel qui donne, dans son étoffe, les moyens de conceptualiser par déplacements, transferts, emprunts ; en transposant, en appliquant, en détournant, en reprenant, etc.; et donc en métaphorisant des représentations et des énoncés qui existent déjà « ailleurs ».

SAVIGNY, BACON, KANT

Ces transferts sont de types divers. Certains peuvent être repérés d'un domaine à un autre, et par exemple du biologique au juridique, comme c'est le cas lorsque Savigny définit et décrit le droit à partir de l'idée de l'organisme du droit (modèle qu'il revendique au départ, comme horizon de recherche, mais dont il ne fera plus usage dans la suite de son travail précis d'historien du droit ${ }^{3}$ ).

Savigny entend définir le droit comme réalité historique, institutionnelle et culturelle : et il lui suffit de suivre une grande évidence d'époque pour poser le droit comme un corps dont l'organisation, la logique, le mode d'existence et la portée sont dessinés dans la notion d'organisme, qui est alors la grande référence cognitive et le type de l'explicateur rationnel. Autrement dit, Savigny recourt à un langage dominant qui fait autorité dans l'ordre du savoir, un langage qui peut donner consistance et légitimité à son propos.

Au niveau de l'exposé, la référence à l'organisme appuie toute une thématique; cette référence conforte de son autorité l'idée que la vie du droit,

3. Friedrich von SAvigny, Vom Beruf unserer Zeit für Gesetzgebung und Rechtswissenschaft, Heidelberg, 1814, notamment p. 8-14. 
au même titre que la langue, la littérature et les mœurs, exprime la vie d'un peuple et la personnalité nationale. Les avantages de cette référence intuitive sont clairs : l'organique permet de penser le juridique dans ses cohésions et ses variations, dans ce qu'il a d'identique à lui-même et de différent selon les lieux et les temps. Le langage de la totalité organique sert aussi de discours de légitimation cognitive, au sens où il pose l'objet traité comme un type d'objet éminemment traitable. Et ce langage fonde, penset-on, la thèse de la prééminence du droit positif sur le droit naturel, puisque le droit positif est la seule réalité socio-historique concrète. Ce sont donc à la fois des avantages conceptuels, épistémiques et partisans ${ }^{4}$.

Inversement, la pensée scientifique et philosophique intègre un bon nombre de métaphores juridiques, ponctuelles ou globales. Bacon et Kant, notamment, placent des jeux d'images juridiques au centre de la description de l'activité scientifique, ou encore au centre de la réflexion philosophique sur le savoir scientifique.

La réflexion de Bacon s'attache longuement au statut du savant et à ses images de rôle. Dans l'utopie de la Nouvelle Atlantide, le savant fait figure de notable institutionnel. En ce sens, le savant est l'héritier des vieux rêves de rôle du philosophe : comme il est devenu le guide capable de multiplier la puissance du prince, il est aussi de ce fait celui qui touche au pouvoir, le conseiller du prince. Mais le statut du savant, chez Bacon, est à la fois traditionnel et futuriste. Il dispose de conditions de recherches idéales dans la Maison de Salomon, cette institution collective merveilleusement équipée dont les membres sont des dignitaires puissants et honorés. On voit d'ailleurs un seigneur de la science passer dans la rue dans un apparat somptueux. C'est un chercheur privilégié et un prince.

Ce qui nous est montré ne correspond bien sûr en rien à la réalité scientifique du premier quart du xviI siècle. C'est déjà le rêve de la science comme suprascience, liée au pouvoir et au prestige du pouvoir - proche aussi des thèmes symboliques de son quasi-contemporain Prospero. Puisque la science est promesse de puissance, le savant est déjà posé comme une figure d'autorité.

Dans le Nouvel Organon, et plus particulièrement dans le chapitre sur l'induction, Bacon présente cette figure d'autorité et de décision comme un juge. Un juge qui est la clé de la situation, certes, mais un juge dont l'activité judiciaire est vraiment surprenante, car il est le seul sujet, et donc le seul point de vue et le seul agent. Ce n'est pas un juge à qui on s'adresse pour résoudre des drames ou des différends; il n'a pas pour fonction de répondre à des problèmes qu'on se pose et qu'on lui pose. Au contraire, c'est lui qui suscite les problèmes, car il a l'entière initiative des cas.

4. J. Schlanger, Les Métaphores de l'organisme, Paris, L'Harmattan, 1995, p. 11-36, 133$140,219-225,255-262$. 
Toute son activité expérimentale diffuse a pour objet d'engendrer des témoignages en tout sens. Il est occupé en permanence à mettre sur pied des expériences pour initier des témoignages et forcer les témoins à comparaître devant lui. Ce qui revient à dire qu'il interfere sans cesse avec le déroulement naturel; il tourmente la nature et trouble le jeu de ses données; il abîme, déchire, ampute, violente les données pour les forcer à porter témoignage et extraire leur aveu. En fait, il passe son temps à torturer les données naturelles qu'il transforme ainsi en témoins. Les expériences multiplient les crissements et les gémissements de la nature et le bavardage de sa souffrance. Le juge-inquisiteur, lui, est à l'écoute du bruit pertinent : il recherche l'aveu qui livre et l'information qui éclaire.

La coupure baconienne entre recensio et judicium, entre la collecte des faits et leur interprétation, entre la donnée et le jugement, l'expérimentation et la théorisation, cette coupure signifie ici que les expériences ne sont pas guidées par une hypothèse préalable. Elles se font en l'air, au hasard, pour troubler, pour déranger, pour voir. En principe, le juge baconien n'a pas de préconceptions et d'hypothèses directrices; c'est pourquoi ses gains restent disparates et il ne peut pas clore ses dossiers.

Ce juge despotique est proche de la grande chasse aux sorcières qui accompagne, pendant la première moitié du Xvil siècle, la naissance de la science. C'est alors que s'élabore, autour de la torture des sorcières, toute une ethnographie du démoniaque. Ce pseudo-savoir fantastique, obtenu par des méthodes "modernes " et « rigoureuses", tous les aveux sous la torture viennent le confirmer et l'élaborer. Le juge baconien, lui aussi, assume l'initiative de l'enquête, la charge méthodologique de sa conduite, et la reconstitution du sens. Il mène une enquête indéterminée, ouverte, violente, qui a pour objet général la recherche de la vérité à partir de l'établissement des faits particuliers. Du reste, cette enquête perpétuelle n'aboutit pas à un procès. Il n'y a d'ailleurs pas de jugement : le juge est actif et même agressif, mais il est muet. C'est un juge qui ne connaît pas la loi, puisque la loi est cachée et dispersée dans la nature. Il veut la découvrir, c'est le but de tous ses efforts, et c'est la nature martyrisée qu'il espère obliger à livrer sa loi.

On sait que cette image judiciaire approximative et même impropre a joué un rôle historique très important dans la formation de l'ethos scientifique. Pour que la métaphore réussisse, il n'était pas nécessaire qu'elle propose une maquette conceptuelle exacte. Pour qu'elle passe au cœur des attitudes épistémiques pendant plusieurs siècles, il n'était pas nécessaire qu'elle décrive convenablement le juge qui lui sert de modèle, ni d'ailleurs le savant qu'elle invente. Une certaine impropriété n'empêche pas l'image d'être porteuse. Il a suffi que la métaphore judiciaire propose à un type nouveau d'activité professionnelle une forme plausible et une image de rôle flatteuse. 
C'est une autre fonction que remplissent les images économiques du succès ou de l'insuccès épistémique, qui sont si voyantes dans la Critique de la raison pure. Ces images sont diffuses, mais particulièrement denses au niveau de la préface, là où Kant, pour décrire la situation actuelle du savoir et le drame auquel va répondre la résolution critique, présente le malheur de la métaphysique par rapport aux autres disciplines. La métaphysique est une reine errante et déchue, malheureuse parmi les autres activités intellectuelles parce qu'elle n'a plus désormais de lieu ni de juridiction qui lui soient propres. Elle se retrouve sans domaine, c'est-à-dire sans territoire et sans propriété; son grand malheur est qu'elle n'a plus de patrimoine à gérer, et qu'elle a perdu la sécurité d'une possession, d'une autorité et d'une activité légitimes.

Son malheur et son désir sont caractérisés avec beaucoup de force en termes de possession et de juridiction. Et si ce langage d'allure juridique est particulièrement dense en ce point, il n'abandonne jamais Kant, qui décrit volontiers l'idéal épistémologique comme la saine gestion qui fera fructifier un patrimoine légitime. Comme on l'a souvent noté, ces termes juridico-économiques recouvrent des notions de père de famille bourgeois. Autrement dit, ces images viennent du droit pour autant que le droit fait partie de la société, de sa réalité et de son univers mental; elles ne viennent pas de la philosophie du droit.

Kant expose la résolution critique du drame initial à travers l'image du tribunal de la raison. Est-ce à dire que la raison se retrouve ici dans le rôle du juge? Au premier abord il semble que oui : on est invité à penser qu'il s'agit d'un procès, puisque les parties en conflit (les positions philosophiques divergentes) demandent un verdict qui les départage et que chacun des candidats espere obtenir l'approbation et l'appui du tribunal. Deux hypothèses scientifiques sont en compétition, deux options symétriques, deux programmes de travail concurrents; à la raison professionnelle d'examiner les arguments, de juger, et de départager les concurrents pour n'en retenir qu'un : c'était aussi la position de Lakatos.

En fait, le tribunal kantien attentif au plaidoyer des deux points de vue opposés est surtout riche d'échos scolaires. Il reprend la mise en scène de la disputatio médiévale, cette forme traditionnelle de l'enseignement supérieur où les positions intellectuelles s'exposent oralement d'une manière conflictuelle, sic et non, et c'est le jugement du public savant, guidé par le jugement du maître, qui décidera et choisira.

Plus encore, cette disputatio n'est pas seulement un grand souvenir fondateur, elle fait partie des projets pédagogiques les plus modernes de 
l'Allemagne de la fin du xviII ${ }^{e}$ siècle ${ }^{5}$. On la retrouve, transposée, dans le célèbre séminaire philologique, celui que Humboldt va instituer officiellement à l'université de Berlin en 1808, mais qui était déjà pratiquée dans quelques universités (dès 1787 , il y avait un séminaire philologique à l'université de Halle et un séminaire pédagogique à l'université de Berlin), de sorte que l'institution du séminaire apparât à la même époque que la Critique. Institution que la France et l'Angleterre envieront longtemps à l'Allemagne, pour l'intégrer enfin comme le couronnement de l'enseignement universitaire défini désormais, vers la fin du $\mathrm{xIx}^{\mathrm{e}}$ siècle, comme un enseignement de recherche. Dans le séminaire philologique à l'allemande, les étudiants de recherche préparent et défendent des interprétations opposées des mêmes textes classiques, soit à l'avance par écrit, soit oralement, et toujours en latin. Le professeur écoute leurs exposés contradictoires sans intervenir; à l'issue, en quelques mots, il opine, il décide, il tranche ${ }^{6}$.

Mais Kant mêle aussi le thème du tribunal de la raison à celui de l'arène de la raison, autre thème médiéval qui a des connotations très différentes. Dans cette arène, la lutte intellectuelle devient un tournoi, ce qui lui donne une dimension cérémonielle et sportive : on se bat, c'est la guerre, mais la guerre ritualisée et euphorisée. C'est le jeu interminable où les combattants se relèvent pour recommencer.

À quoi s'adjoint un autre écho médiéval, celui de l'épreuve : le jugement de Dieu récompense le vainqueur légitimé. Que le meilleur gagne! En fait, la raison critique, telle que la décrit Kant, remplit moins un rôle de juge qu'un rôle d'arbitre : sa fonction est de veiller aux conditions du champ, de donner le départ du combat, d'en garantir la correction, d'en proclamer l'issue. En somme, la raison tient ici le rôle de l'État libéral, celui qui, par définition, garantit les conditions $d w$ champ et son bon fonctionnement, mais ne se mêle pas des enjeux et n'intervient pas dans la lutte ${ }^{7}$. C'est de cette façon que Mirabeau, en 1791, voit dans l'État un pur arbitre en matière d'enseignement et de savoir :

« Tout homme a le droit d'enseigner ce qu'il sait, et même ce qu'il ne sait pas [...] Enseigner est un genre de commerce : le vendeur s'efforce de faire valoir sa marchandise; l'acheteur la juge, et tâche de l'obtenir au plus bas prix : le pouvoir public, spectateur et garant du marché, ne saurait y prendre part [...] il

5. R. Steven TurNer, « Historicism, Kritik, and the Prussian Professorate, 1790 to 1840 », in Philologie et Herméneutique au xix' siècle, II, éd. par Mayotte Bollack et Heinz WiSmanN, Göttingen, Vanderhoeck \& Ruprecht, 1983, p. 462-464.

6. Victor Cousin, Rapport sur l'Instruction publique dans quelques pays de l'Allemagne, 1831, Euvres, Bruxelles, 1840-1841, t. III, p. 31-33 et 51-52.

7. Wilhelm von Humbold, Ideen zu einem Versuch die Grenzen der Wirksamkeit des Staats zu bestimmen, rédigé (mais non publié sous forme de livre) en 1791-1792. 
n'est là que pour les laisser tous agir librement, et pour les maintenir en paix $\gg^{8}$.

La raison critique est un juge libéral qui a les mêmes obligations et les mêmes limites que l'État libéral.

Ainsi la raison juge est devenue le juge professeur et le juge arbitre. On peut se demander pourquoi Kant a recours à ces métaphores dont le point est différent et qui glissent l'une dans l'autre non sans flou. Il a probablement besoin de ce bouquet d'images pour gagner son propre point de vue, pour penser sa pensée et l'exposer. Ce ne sont pas là des métaphores qui ont pour objet de décorer, d'orner, d'ajouter; des métaphores dont on pourrait se passer et qu'on pourrait éliminer sans perte en formalisant le raisonnement. Elles ont pour fonction de porter et de nourrir une intuition neuve, différente, qui a besoin de moyens et de formes pour commencer à se conceptualiser et donc aussi à s'énoncer. Ce que Kant cherche à dire est assez déroutant, et sans doute pour lui-même aussi. Il a besoin de repères culturels pour rendre son propos plus facile, plus familier et donc plus saisissable. Il n'est pas étonnant qu'un livre novateur comme la Critique baigne dans un enchevêtrement d'allusions métaphoriques tantôt voisines (avoir un bien propre à gérer, la raison comme le professeur du séminaire ou comme l'État libéral), tantôt archaïques (la joute et l'épreuve). Sans doute faut-il parler avec du même pour pouvoir penser du neuf et du différent.

\section{FÉCONDITÉ DU MÉTAPHORIQUE}

Ces exemples choisis entre tant d'exemples possibles renvoient à la question plus générale du recours aux métaphores. On voit que la réflexion inventive s'organise volontiers autour de figures notionnelles qu'elle reprend pour son usage, et que la pensée qui se cherche a très souvent recours à des images directrices empruntées à des noyaux de langage qui viennent d'ailleurs. C'est une démarche tout à fait courante et par là même importante.

L'investissement métaphorique de ce qu'on entreprend de connaître suppose avant tout que, quel que soit le problème intellectuel qui nous absorbe à un moment donné, il y ait toujours aussi un « ailleurs », ou plutôt des « ailleurs ». Par-delà la question qui est au centre de l'attention, beaucoup

8. Bronislaw Baczko, Une Éducation pour la démocratie. Textes et projets de l'époque révolutionnaire, Paris, Garnier, 1982, p. 73. 
d'autres subsistent à l'horizon. L'horizon intellectuel et culturel reste présent comme un plein riche, dénivelé, multiple; un plein où l'on peut circuler parmi des organisations du sens: des termes, des vocables, des notions, des aspects ou des moules de discours. Tout un environnement intellectuel-culturel donne des manières de dire et fournit de quoi parler. On peut dire que ce qui donne accès au potentiel métaphorique, c'est d'abord que la vie intellectuelle est une vie plurielle, de sorte que l'intelligence baigne dans la pluralité. Une information souvent informe, mais démultipliée, maintient plusieurs types de réservoirs sémantiques.

On peut énumérer très rapidement : a) le langage courant, chargé de notions d'origine juridique, religieuse, etc., qui sont souvent des métaphores endormies; b) les langages spécialisés, érudits, ésotériques, techniques, professionnels, etc. Ceux-là présentent l'attrait du rare face à l'attrait, non moins puissant, du familier; c) le langage cognitif dominant de l'époque, ce langage surévalué devenu modèle de la réussite rationnelle et source de métaphores légitimantes : ce qui a été le cas, notamment, pour le langage de l'organisme et de l'organisation, pour le langage de la structure, plus récemment pour le langage de l'Intelligence Artificielle; d) tout un répertoire culturel de thèmes imagés récurrents : l'horloge, la balance, la vie organique, l'harmonie, la vision, le grand thêâtre du monde, etc. Ce stock métaphorique accompagne la pensée occidentale tout au long de son histoire, alors que, pour chaque thème, chaque vague d'usage est différente : si bien que chaque reprise continue et nourrit la lignée tout en servant de support à un autre argument.

On notera que le recours à tel ou tel langage métaphorique, comme aussi son succès, ne dépendent pas simplement de sa disponibilité. Il ne s'agit pas de puiser à volonté, en fonction des besoins, dans une donne neutre également disponible. Il est très visible, au contraire, que certains langages s'imposent à un moment donné comme l'autorité même du rationnel. Quant au succès intellectuel d'un langage métaphorique, il est directement lié à la conjoncture, c'est-à-dire aux enjeux intellectuels spécifiques qui organisent le sentiment de pertinence, et aux enjeux intellectuels d'époque qui rapprochent certains possibles en les valorisant comme évidents.

C'est un dispositif heuristique où l'impureté peut être féconde et l'impropriété constitutive. En effet, l'usage métaphorique d'une notion ou d'un ensemble de termes a nécessairement quelque chose d'approximatif, qui ne répond pas à l'idéal d'une méthode rigoureuse et d'une saine logique. Les modèles métaphoriques ne sont que métaphoriquement modèles. D'une part, le métaphorique retient à sa convenance quelques traits dérivés du modèle d'origine, sans se soucier de le rendre exactement; il filtre l'argument en déformant la source. D'autre part, il pose, nomme, caractérise par anticipation quelque chose d'inédit qu'on ne connait pas encore et dont on ne peut pas encore parler avec pertinence. 
Et pourtant, l'intuition métaphorique joue un rôle décisif dans la formation du discours de la connaissance. En balisant grossièrement l'espace, le métaphorique fait surgir le champ comme tel, et donc le rend nommable et abordable. C'est une première prise de possession. D'ailleurs, cette prise métaphorique ne disparaît pas comme un échafaudage qu'on retire dès qu'on n'en a plus besoin. Comme ses catégories intuitives ne sont pas neutres, elles orientent la suite en façonnant le relief du paysage et le centrage de l'attention.

En outre, les nouvelles approches, les tournants, les réinterprétations en cours de route, les progrès théoriques, bref tout ce qui tient à l'avancée de la pensée et aux transformations d'une connaissance sans inertie et sans repos, tous ces déplacements successifs n'échappent pas non plus au métaphorique. Vision, démarche, modèle, ou plus simplement image ou constellation de termes : dans l'activité de connaissance, les modifications sont permanentes, qu'il s'agisse de renouvellements à grande échelle ou de rajustements minimes. De sorte que la fonction heuristique du métaphorique n'est pas simplement un premier échafaudage plutôt vague, mais temporaire, que la suite, plus rigoureuse, oubliera. C'est tout au long de la vie théorique d'une discipline que l'intuition métaphorique renouvelle la pensée connaissante, en colorant les catégories qu'elle apporte et qu'elle soutient.

On pourrait conclure en soulignant qu'il est souhaitable d'avoir des goûts intellectuels variés et beaucoup de curiosités et de champs d'intérêts, pour nourrir le virtuel, le surplus, le pluriel, et enrichir cet horizon culturel de la mémoire qui est une des ressources de la créativité. Ce serait une conclusion peu contestée, mais sans surprise : personne ne s'étonnera de l'idée qu'un esprit cultivé a plus de chances d'être fécond.

Je préfêre conclure en évoquant la contrepartie de la fécondité, qui est le risque : le risque que court une nouvelle entreprise de pensée qui naît à l'écart, dans la divergence, en proposant un cadre de pensée différent pour dire quelque chose d'autre qui ne se trouve pas ailleurs. Ce risque est réel, car tout ce qui est énoncé et proposé n'est pas accepté. Non seulement tout n'est pas intégré comme fécond, mais encore tout n'est pas reçu comme raisonnable. Il existe aussi une production d'idées qu'on appelle déviante; celle-là est maintenue à l'extérieur de la communauté scientifique et rejetée hors du champ de la raison.

Or l'état naissant de la réflexion, cette situation de la naissance prise dans son flou et ses imprudences, ne permet pas de reconnaître sur-lechamp dans quel cas on se trouve, et de savoir si on est seul temporairement parce qu'on est le premier à frayer une voie que l'adhésion générale rejoindra par la suite; ou si on est seul essentiellement, parce que ce qu'on propose est jugé trop étrange, et paraît si clairement aberrant et stérile que 
nul ne va le considérer ou le tester. Si bien que la solitude du penseur qui élabore un point de vue vraiment différent comporte effectivement un risque rationnel; non seulement le risque de l'échec et de l'erreur (vingt ans perdus dans un mauvais investissement, ce qui est déjà assez lourd!), mais aussi le risque du délire et de la démence. Ce risque fait la substance des rêves du jeune Descartes : s'il voit d'une évidence aveuglante une perspective inédite radicale, comment juger cet enthousiasme et cette « ambition incroyable »? Comment être sûr qu'il peut faire fond sur son propre jugement $^{9}$ ? La question se pose beaucoup moins fortement, bien sûr, lorsqu'on travaille (comme c'est le cas le plus souvent) sur un problème déjà reconnu dans un cadre déjà donné. Mais lorsqu'on introduit un point de vue qui altère le paysage et qui transforme le cadre ou la perspective, l'insécurité reprend toute son acuité et toute sa justesse. La pensée connaissante à l'état naissant n'a pas de garanties, sinon des assurances circonstantielles (de nos jours, être intégré dans un cadre professionnel reconnu, par exemple). Et la vision la plus novatrice est la moins garantie.

$\mathrm{Si}$ je termine en mentionnant le risque, ce n'est pas pour assombrir par la menace d'un danger, ni d'ailleurs pour appeler à la prudence. C'est parce que ce risque marque justement l'importance intellectuelle et rationnelle de l'invention métaphorique. Si l'échec peut être grave et le désastre radical, c'est bien la contrepartie de la fécondité du métaphorique. C'est le revers du fait que, globalement, dans son impureté même, l'entreprise de la pensée connaissante réussit.

Judith SCHLANGER

(juillet 1992).

9. Henri GouHrer, Essais sur Descartes, Paris, Vrin, 1937, p. 47-59. 